\title{
Estimation of unknown function of a class of integral inequalities with pth power
}

\author{
Yusong Lu ${ }^{a}$, Wu-Sheng Wang ${ }^{b}$, Hua Zhong ${ }^{c^{*}}$ \\ School of Mathematics and Statistics, Hechi University, Guangxi, Yizhou 546300, P. R. China \\ aluyusong@yeah.net, 'wang4896@126.com, asonfan@163.com
}

Keywords: Integral inequality; Integral inequality technique; Modified Riemann-Liouville fractional derivative; Riemann-Liouville fractional integral; Estimation of solution.

\begin{abstract}
Fractional differential equations and fractional integral equations have gained considerable importance and attention due to their applications in many engineering and scientific disciplines. Gronwall-Bellman inequalities are important tools in the study of existence, uniqueness, boundedness, stability and other qualitative properties of solutions of Fractional differential equations and fractional integral equations. In this paper, we discuss a class of integral inequalities with pth power, which includes a nonconstant term outside the integrals. Using the definitions and properties of modified Riemann-Liouville fractional derivative and Riemann-Liouville fractional integral, the upper bounds of the unknown function is estimated explicitly. The derived result can be applied in the study of qualitative properties of solutions of fractional integral equations.
\end{abstract}

\section{Introduction}

Fractional differential equations and fractional integral equations have gained considerable importance and attention due to their applications in many engineering and scientific disciplines. Gronwall-Bellman inequalities [1,2] are important tools in the study of existence, uniqueness, boundedness, stability, invariant manifolds and other qualitative properties of solutions of fractional differential equations and fractional integral equations. In 2011, Abdeldaim et al. [3] studied a new iterated integral inequality with pth power

$$
u(t) \leq u_{0}+\int_{0}^{t} f(s) u(s)\left[u(s)+\int_{0}^{s} h(\tau)\left[u(\tau)+\int_{0}^{\tau} g(\xi) d \xi\right] d \tau\right] d s .
$$

In 2014, El-Owaidy, Abdeldaim, and El-Deeb [4] discussed a new nonlinear integral inequality with a nonconstant term outside the integrals

$$
u(t) \leq f(t)+\int_{a}^{t} g(s) u^{p}(s) d s+\int_{a}^{\alpha(t)} h(s) u^{p}(s) d s .
$$

In 2014, Zheng [5] investigated the inequality with fractional integral

$$
u(t) \leq C+\frac{1}{\Gamma(\alpha)} \int_{0}^{t}(t-s)^{\alpha-1} g(s) u(s) d s+\frac{1}{\Gamma(\alpha)} \int_{0}^{T}(T-s)^{\alpha-1} g(s) u(s) d .
$$

In order to achieve various goals, some investigators have established a lot of useful and interesting integral inequalities (see [6-10] and the references cited therein).

In this paper, on the basis of $[3,4,5]$, we discuss a class of integral inequality with pth power

$$
u(t) \leq f(t)+\frac{1}{\Gamma(\alpha)} \int_{0}^{t}(t-s)^{\alpha-1} g(s)\left[u(s)+\frac{1}{\Gamma(\alpha)} \int_{0}^{s}(s-\tau)^{\alpha-1} h(\tau) u(\tau) d \tau\right]^{p} d s .
$$

\section{Result}

Throughout this paper, let $R_{+}=[0,+\infty)$.

Jumarie [7, 8] given the following definitions for the modified Riemann-Liouville fractional derivative and fractional integral. 
Definition 1. The modified Riemann-Liouville derivative of order $\alpha$ is defined by the following expression:

$$
D_{t}^{\alpha} f(t)=\left\{\begin{array}{l}
\frac{1}{\Gamma(1-\alpha)} \frac{d}{d t} \int_{0}^{t}(t-\xi)^{-\alpha}(f(\xi)-f(0)) d \xi, 0<\alpha<1, \\
\left(f^{(n)}(t)\right)^{(\alpha-n)}, n<\alpha<n+1, n \geq 1 .
\end{array}\right.
$$

Definition 2.The Riemann-Liouville fractional integral of order $\alpha$ on the interval is defined by

$$
I^{\alpha} f(t)=\frac{1}{\Gamma(1+\alpha)} \int_{0}^{t} f(s)(d s)^{\alpha}=\frac{1}{\Gamma(\alpha)} \int_{0}^{t}(t-s)^{\alpha-1} f(s) d s .
$$

In 2014, Zheng [5] proved the following property for the modified Riemann-Liouville derivative and fractional integral.

Suppose that $0<\alpha<1, f$ is a continuous function, then

$$
D_{t}^{\alpha}\left(I^{\alpha} f(t)\right)=f(t) \text {. }
$$

Some important properties for the modified Riemann-Liouville derivative and fractional integral are listed as follows (see $[9,10])$ :

$$
\begin{aligned}
& D_{t}^{\alpha} t^{r}=\frac{\Gamma(1+r)}{\Gamma(1+r-\alpha)} t^{r-\alpha}, \\
& D_{t}^{\alpha}(f(t) g(t))=g(t) D_{t}^{\alpha} f(t)+f(t) D_{t}^{\alpha} g(t) . \\
& D_{t}^{\alpha} f[g(t)]=f_{g}^{\prime}[g(t)] D_{t}^{\alpha} g(t)=D_{t}^{\alpha} f\left[g(t)\left(g^{\prime}(t)\right)^{\alpha},\right. \\
& I^{\alpha}\left(D_{t}^{\alpha} f(t)\right)=f(t)-f(0), \\
& D_{t}^{\alpha} C=0, \text { where } C \text { is a constant. }
\end{aligned}
$$

Theorem 1. Suppose that $g(t), h(t) \in C\left(R_{+}, R_{+}\right), f \in\left(R_{+}, R_{+}\right)$is a nondecreasing function with $f$ $(t)>0$ for $t>0$, and

$$
1-\frac{1}{\Gamma(\alpha)} \int_{0}^{t}(t-\tau)^{\alpha-1} \exp \left[-\int_{0}^{\frac{\tau^{\alpha}}{\Gamma(1+\alpha)}} p h\left((s \Gamma(1+\alpha))^{\frac{1}{\alpha}}\right) d s\right] p g(\tau) f^{p}(\tau) d \tau>0 .
$$

If $u(t)$ satisfies the inequality (4), then

$$
\begin{aligned}
u(t) \leq & f(t) \exp \left[-\int_{0}^{\frac{t^{\alpha}}{\Gamma(1+\alpha)}} h\left((s \Gamma(1+\alpha))^{\frac{1}{\alpha}}\right) d s\right] \\
& \times\left\{1-\frac{1}{\Gamma(\alpha)} \int_{0}^{t}(t-\tau)^{\alpha-1} \exp \left[-\int_{0}^{\frac{\tau^{\alpha}}{\Gamma(1+\alpha)}} p h\left((s \Gamma(1+\alpha)) \frac{1}{\alpha}\right) d s\right] p g(\tau) f^{p}(\tau) d \tau\right\}^{-1 / p} .
\end{aligned}
$$

Proof. Since $\mathrm{f}(\mathrm{t})$ is a positive and nondecreasing function. The inequality (4) can be rewritten to

$$
\frac{u(t)}{f(t)} \leq 1+\frac{1}{\Gamma(\alpha)} \int_{0}^{t}(t-s)^{\alpha-1} g(s) f^{p}(s) \frac{u(s)}{f(s)}\left[\frac{u(s)}{f(s)}+\frac{1}{\Gamma(\alpha)} \int_{0}^{s}(s-\tau)^{\alpha-1} h(\tau) \frac{u(\tau)}{f(\tau)} d \tau\right]^{p} d s .
$$

Let $z_{l}(t)=u(t) / f(t)$. From $(16)$ we get that

$$
z_{1}(t) \leq 1+\frac{1}{\Gamma(\alpha)} \int_{0}^{t}(t-s)^{\alpha-1} g(s) f^{p}(s) z_{1}(s)\left[z_{1}(s)+\frac{1}{\Gamma(\alpha)} \int_{0}^{s}(s-\tau)^{\alpha-1} h(\tau) z_{1}(\tau) d \tau\right]^{p} d s .
$$

Define a function $z_{2}(t)$ by the right hand side of the above inequality. Then $z_{2}(t)$ is a positive and nondecreasing function on $R_{+}$. Obviously, we have

$$
z_{1}(t) \leq z_{2}(t), t \in R_{+}, \quad z(0)=1
$$

By use of (7) and (17) we have

$$
D_{t}^{\alpha} z_{2}(t)=g(t) f^{p}(t) z_{1}(t)\left[z_{1}(t)+\frac{1}{\Gamma(\alpha)} \int_{0}^{t}(t-\tau)^{\alpha-1} h(\tau) z_{1}(\tau) d \tau\right]^{p}
$$




$$
\leq g(t) f^{p}(t) z_{2}(t)\left[z_{2}(t)+\frac{1}{\Gamma(\alpha)} \int_{0}^{t}(t-\tau)^{\alpha-1} h(\tau) z_{2}(\tau) d \tau\right]^{p}=g(t) f^{p}(t) z_{2}(t) z_{3}^{p}(t),
$$

where

$$
z_{3}(t)=z_{2}(t)+\frac{1}{\Gamma(\alpha)} \int_{0}^{t}(t-\tau)^{\alpha-1} h(\tau) z_{2}(\tau) d \tau,
$$

From (18) and (19), we have

$$
D_{t}^{\alpha} z_{3}(t)=D_{t}^{\alpha} z_{2}(t)+h(t) z_{2}(t) \leq g(t) f^{p}(t) z_{2}(t) z_{3}{ }^{p}(t)+h(t) z_{2}(t) \leq g(t) f^{p}(t) z_{3}(t) z_{3}{ }^{p}(t)+h(t) z_{3}(t) .
$$

Let $x(t)=-\left(z_{3}(t)\right)^{-p}$, then $x(0)=-\left(z_{3}(0)\right)^{-p}=-1$, from (20) we get

$$
D_{t}^{\alpha} x(t)-p h(t) x(t) \leq p g(t) f^{p}(t) \text {. }
$$

On other hand, by the properties (8), (9), (10), we have

$$
D_{t}^{\alpha}\left\{x(t) \exp \left[-\int_{0}^{\frac{t^{\alpha}}{\Gamma(1+\alpha)}} p h\left((s \Gamma(1+\alpha))^{\frac{1}{\alpha}}\right) d s\right]\right\} \leq \exp \left[-\int_{0}^{\frac{t^{\alpha}}{\Gamma(1+\beta)}} p h\left((s \Gamma(1+\alpha))^{\frac{1}{\alpha}}\right) d s\right] p g(t) f^{p}(t) .
$$

Substituting $t$ with $\tau$ in (22), making a fractional integral of order $\alpha$ for (22) with respect to $\tau$ from 0 to $t$ and using the properties (7), we get that

$$
\begin{aligned}
& x(t) \exp \left[-\int_{0}^{\frac{t^{\alpha}}{\Gamma(1+\alpha)}} \operatorname{ph}\left((s \Gamma(1+\alpha))^{\frac{1}{\alpha}}\right) d s\right]-x(0) \\
& \leq \frac{1}{\Gamma(\alpha)} \int_{0}^{t}(t-\tau)^{\alpha-1} \exp \left[-\int_{0}^{\frac{\tau^{\alpha}}{\Gamma(1+\alpha)}} p h\left((s \Gamma(1+\alpha))^{\frac{1}{\alpha}}\right) d s\right] p g(\tau) f^{p}(\tau) d \tau .
\end{aligned}
$$

By the relation $x(t)=-\left(z_{3}(t)\right)^{-p}$, from (23) we have

$$
\begin{aligned}
& z_{3}(t) \leq \exp \left[-\frac{1}{p} \int_{0}^{\frac{t^{\alpha}}{\Gamma(1+\alpha)}} p h\left((s \Gamma(1+\alpha))^{\frac{1}{\alpha}}\right) d s\right] \\
& \quad \times\left\{1-\frac{1}{\Gamma(\alpha)} \int_{0}^{t}(t-\tau)^{\alpha-1} \exp \left[-\int_{0}^{\frac{\tau^{\alpha}}{\Gamma(1+\alpha)}} p h\left((s \Gamma(1+\alpha))^{\frac{1}{\alpha}}\right) d s\right] p g(\tau) f^{p}(\tau) d \tau\right\}^{-1 / p} .
\end{aligned}
$$

From the relations $u(t) / f(t)=v(t) \leq z(t) \leq w(t)$. We get the required estimation (14). The proof is complete.

\section{Summary}

In this paper, we discuss a class of integral inequalities with pth power

$$
u(t) \leq f(t)+\frac{1}{\Gamma(\alpha)} \int_{0}^{t}(t-s)^{\alpha-1} g(s)\left[u(s)+\frac{1}{\Gamma(\alpha)} \int_{0}^{s}(s-\tau)^{\alpha-1} h(\tau) u(\tau) d \tau\right]^{p} d s .
$$

Under the condition

$$
1-\frac{1}{\Gamma(\alpha)} \int_{0}^{t}(t-\tau)^{\alpha-1} \exp \left[-\int_{0}^{\frac{\tau^{\alpha}}{\Gamma(1+\alpha)}} p h\left((s \Gamma(1+\alpha))^{\frac{1}{\alpha}}\right) d s\right] p g(\tau) f^{p}(\tau) d \tau>0,
$$

we obtain the upper bounds of the embedded unknown function $u(t)$

$$
\begin{aligned}
u(t) & \leq f(t) \exp \left[-\int_{0}^{\frac{t^{\alpha}}{\Gamma(1+\alpha)}} h\left((s \Gamma(1+\alpha))^{\frac{1}{\alpha}}\right) d s\right] \\
& \times\left\{1-\frac{1}{\Gamma(\alpha)} \int_{0}^{t}(t-\tau)^{\alpha-1} \exp \left[-\int_{0}^{\frac{\tau^{\alpha}}{\Gamma(1+\alpha)}} p h\left((s \Gamma(1+\alpha))^{\frac{1}{\alpha}}\right) d s\right] p g(\tau) f^{p}(\tau) d \tau\right\}^{-1 / p},
\end{aligned}
$$


by adopting inequality techniques. The derived result can be applied in the study of qualitative properties of solutions of fractional integral equations.

\section{Acknowledgement}

This research was supported by Guangxi Natural Science Foundation (Project No. 2012GXNSFAA053009) and Scientific Research Foundation of the Education Department of Guangxi Autonomous Region of China (No. KY2015ZD103, KY2015YB257). The corresponding author is Hua Zhong.

\section{References}

[1] T.H. Gronwall, Note on the derivatives with respect to a parameter of the solutions of a system of differential equations, Ann Math. 20 (1919)292-296.

[2] R. Bellman, The stability of solutions of linear differential equations, Duke Math J. 10 (1943) 643-647.

[3] A. Abdeldaim and M. Yakout, On some new integral inequalities of Gronwall-Bellman-Pachpatte type, Appl Math Comput. 217(2011)7887-7899.

[4] H. El-Owaidy, A. Abdeldaim and A. A. El-Deeb, On some new retarded nonlinear integral inequalities and theirs Applications, Mathematical Sciences Letters, 3(3)(2014)157-164.

[5] B. Zheng, Explicit bounds derived by some new inequalities and applications in fractional integral equations, Journal of Inequalities and Applications, 2014(4)(2014)1-12.

[6] B. G.Pachpatte, Inequalities for Differential and Integral Equations, London, Academic Press, 1998.

[7] G. Jumarie, Modified Riemann-Liouville derivative and fractional Taylor series of nondifferentiable functions further results, Comput. Math. Appl. 51(2006)1367-1376.

[8] G. Jumarie, Table of some basic fractional calculus formulae derived from a modified RiemannLiouville derivative for non-differentiable functions,Appl. Math. Lett. 22 (2009)378-385.

[9] GC . Wu, and EWM. Lee, Fractional variational iteration method and its application. Phys. Lett. A 374, (2010)2506-2509

[10] B. Zheng, (G'/G)-expansion method for solving fractional partial differential equations in the theory of mathematical physics. Commun. Theor. Phys. 58(2012)623-630. 